\title{
Hybrid simulations: combining atomistic and coarse-grained force fields using virtual sites
}

\author{
Andrzej J. Rzepiela, ${ }^{a}$ Martti Louhivuori, ${ }^{a}$ Christine Peter ${ }^{* b}$ and \\ Siewert J. Marrink*a
}

\begin{abstract}
Hybrid simulations, in which part of the system is represented at atomic resolution and the remaining part at a reduced, coarse-grained, level offer a powerful way to combine the accuracy associated with the atomistic force fields to the sampling speed obtained with coarse-grained (CG) potentials. In this work we introduce a straightforward scheme to perform hybrid simulations, making use of virtual sites to couple the two levels of resolution. With the help of these virtual sites interactions between molecules at different levels of resolution, i.e. between CG and atomistic molecules, are treated the same way as the pure $\mathrm{CG}-\mathrm{CG}$ interactions. To test our method, we combine the Gromos atomistic force field with a number of coarse-grained potentials, obtained through several approaches that are designed to obtain CG potentials based on an existing atomistic model, namely iterative Boltzmann inversion, force matching, and a potential of mean force subtraction procedure (SB). We also explore the use of the MARTINI force field for the CG potential. A simple system, consisting of atomistic butane molecules dissolved in CG butane, is used to study the performance of our hybrid scheme. Based on the potentials of mean force for atomistic butane in CG solvent, and the properties of 1:1 mixtures of atomistic and CG butane which should exhibit ideal mixing behavior, we conclude that the MARTINI and SB potentials are particularly suited to be combined with the atomistic force field. The MARTINI potential is subsequently used to perform hybrid simulations of atomistic dialanine peptides in both CG butane and water. Compared to a fully atomistic description of the system, the hybrid description gives similar results provided that the dielectric screening of water is accounted for. Within the field of biomolecules, our method appears ideally suited to study e.g. protein-ligand binding, where the active site and ligand are modeled in atomistic detail and the rest of the protein, together with the solvent, is coarse-grained.
\end{abstract}

\section{Introduction}

Molecular dynamics (MD) is a well established technique to simulate the structure and dynamics of soft matter in general, and of biomolecular systems in particular. ${ }^{1-3}$ However, the huge computational effort involved in conventional all-atom (AA) MD simulations currently limits accessible time and length scales. To overcome these limitations, coarse grained (CG) models, in which several atoms are grouped together into effective interaction sites (CG beads), are currently being developed by many groups (for an overview, see ref. 3-8). The sampling efficiency is increased up to several orders of

\footnotetext{
${ }^{a}$ Groningen Biomolecular Sciences and Biotechnology Institute \&

Zernike Institute for Advanced Materials, University of Groningen,

Nijenborgh 7, 9747 AG Groningen, The Netherlands.

E-mail: s.j.marrink@rug.nl; Tel: +31-50-3634457

${ }^{b}$ Max Planck Institute for Polymer Research Ackermannweg 10,

55128 Mainz,Germany.E-mail:peter@mpip-mainz.mpg.de,

Tel: +49 (0)6131379267
}

magnitude with these CG models. Nevertheless, this large gain comes at the cost of a reduced accuracy, due to the inherent simplifications. Thus, in many instances it would be advantageous to combine high resolution $\mathrm{AA}$ and low resolution $\mathrm{CG}$ descriptions in a multiscale approach. Indeed, several of such hybrid schemes have recently been proposed, falling into four classes: back-mapping methods, interface methods, "constant $\lambda$ " techniques and fixed resolution methods. Back mapping methods $^{9-17}$ allow switching between the different levels of resolution, i.e. reconstruct the atomistic structural ensemble that underlies a $\mathrm{CG}$ representation for interesting configurations. In the interface methods ${ }^{18-20}$ molecules change their level of description on the fly when moving between spatially localized high resolution domains and the lower resolution surrounding. With the "constant $\lambda$ " techniques, ${ }^{21-23}$ molecules are described constantly on both levels of resolution, which are mixed with a specified ratio. This approach is especially useful in Hamiltonian exchange simulations, in which the different $\lambda$ levels are coupled. In the last class of methods molecules 


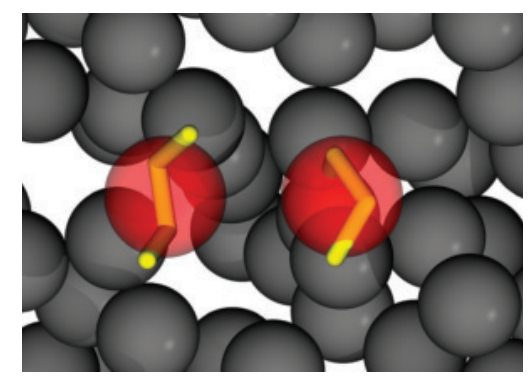

Fig. 1 Hybrid model using virtual sites. Coarse grained beads are represented as gray spheres. Atomistic molecules are depicted as yellow sticks and virtual interaction sites are colored red. Atomistic molecules interact with the low resolution environment through virtual sites and with each other as in a standard atomistic system. Coarse grain beads interact both with each other and with the atomistic components via $\mathrm{CG}$ potentials.

(or parts of molecules) at different levels of resolution exist in the same system, interacting with each other, and the resolution of a given molecule remains constant during the simulation, resembling the idea behind $\mathrm{QM} / \mathrm{MM}$ methods. ${ }^{24}$ Interactions between sites of different resolution in such a multiscale system can be defined with special mixing rules, ${ }^{25}$ or through complete reparameterization of all AA-CG cross interactions. ${ }^{26}$ In the work of Neri et al., ${ }^{27}$ only a protein system is considered with an explicit interface region located between the AA and CG parts in order to bridge the discontinuity between the two models. Further background on the emerging field of multiscale modeling methods can be found in recent reviews. 5,28

In this contribution we present a simple hybrid model, which belongs to the fourth class of multiscale methods implying we consider a system in which a predefined part is represented in full detail, and the remainder at the CG level. The coupling is provided by the atomistic molecules which carry additional, virtual interaction sites, through which they interact with the CG surroundings using standard CG potentials. The simplicity is the main advantage of our model, making it possible to use it with generic CG and AA forcefields and standard simulation packages. Our approach is therefore more general and requires only few additional parameters. However, mixing of resolutions is not trivial. For instance, it remains to be seen how the interaction between two atomistic molecules is affected by the surrounding of a CG solvent compared to the "normal" atomistic surrounding. The expectation is that "multiscale" CG models, i.e. CG models that are directly derived from the atomistic model, are particularly suited for such a hybrid method. Here we test the performance of our hybrid method, combining the GROMOS 53a5/6 atomistic force field and several CG representations: either a heuristic MARTINI potential or "multiscale" CG potentials obtained using the iterative Boltzmann inversion method (IB), force matching (FM), or the subtraction method (SB). We study the performance of the model on two systems, namely liquid butane and dialanine dissolved in butane or water. We show that the simple hybrid model can connect the two resolution scales, most seamlessly with the SB method. Efficient resolution mixing is also observed with the MARTINI model.
The rest of this article is organized as follows. We set out to describe the methodological basis of the hybrid model. Subsequently, we introduce the principles of the applied methods to derive the CG potentials, and provide a detailed description of the simulation and analysis procedures. We proceed with a presentation of the results on the performance of the hybrid model, first for the pure butane system and next for the peptide/solvent systems. A short conclusive section ends this article.

\section{Methods}

\section{A Hybrid model}

In the hybrid model, essential parts of the system, for example solute molecules, are represented at a detailed atomistic resolution, while the remaining elements, for example solvent molecules, are modeled using a lower, coarse grained resolution. An example of a hybrid system consisting of atomistic butane solutes solvated by coarse-grained butanes is depicted in Fig. 1. Both interactions between solvent-solvent pairs and between solvent-solute pairs of molecules are described with the potentials from a low resolution $\mathrm{CG}$ forcefield while the remaining interactions, that is interactions between atomistic molecules, are computed in full detail. To couple an atomistic molecule with a coarse grained environment so called virtual sites are defined, positioned at the center of mass of the group of atoms they represent ( $c f$. Fig. 1). This positioning of the virtual sites corresponds to the mapping scheme between $\mathrm{CG}$ and atomistic models. The force $F_{i}$ from CG interactions acting on the virtual site $i$ is spread over the corresponding atoms, according to the equation

$$
f_{\gamma}=\frac{m_{\gamma}}{M_{i}} F_{i} \quad \forall \gamma \in N_{i}
$$

where $M_{i}=\sum_{\gamma \in N_{i}} m_{\gamma}$ is the total mass of the atomic cluster $N_{i}$ constituting the virtual site, $m_{\gamma}$ is the mass of the $\gamma$ th atom, and $f_{\gamma}$ is the force acting on it. Important features of our scheme are the following: (i) no specific interactions between AA and CG beads need to be parameterized as is done in other boundary methods, ${ }^{26}$ making the method easily applicable to any system of interest, (ii) no position dependent resolution transformation is required, therefore a well defined Hamiltonian exists without violating Newton's 3rd law, two conditions not met with other, dynamic exchange, approaches, ${ }^{29}$ and (iii) the method naturally combines the advantages of FG models (accurate description of the molecule, or part of molecule, of interest) and CG models (explicit treatment of the surroundings at a speed 2-3 orders of magnitude larger compared to AA models). Furthermore, the proposed method is general in the sense that it can, in principle, combine any two force fields provided that the molecule of interest is parametrized at both levels of resolution.

\section{B Simulation setup}

To construct the CG butane-butane potentials (see below), reference simulations of pure butane were performed at the AA level. This system consisted of 750 butane molecules. Corresponding CG simulations were carried out in systems 
of the same size. To test the hybrid model, simulations were performed for two types of systems, namely a pure butane system and a mixture of dialanine and either water or butane. For the butane system, the set-up consisted of (i) two AA butanes dissolved in a box of $748 \mathrm{CG}$ butane (calculation of association constants) or (ii) a box consisting of 375 AA butanes and $375 \mathrm{CG}$ butane molecules (calculation of solvation parameters). For the dialanine containing systems, phase separation was studied in a box containing 370 AA dialanine molecules mixed with $1120 \mathrm{CG}$ solvent beads. For the calculation of the interaction energy between two dialanine peptides, a system consisting of two dialanines and 1050 solvent beads was additionally simulated. Fully atomistic systems were also simulated for reference.

All simulations were performed using the GROMACS simulation package ${ }^{30}$ (version 4.0.5). Atomistic simulations and atomistic parts of hybrid systems were represented with the 53a5/6 parameter set of the GROMOS forcefield. ${ }^{31}$ For the pure butane systems, different CG potentials were used as explained below. The systems containing dialanine peptides were only simulated using the MARTINI CG potentials (version $2.0^{32}$ ). All of the simulations were performed in a periodic cubic box with dimensions longer than twice the cut-off distance. A cut-off distance for nonbonded interactions was set at $1.4 \mathrm{~nm}$ for pure butane systems and $1.2 \mathrm{~nm}$ (MARTINI potentials) or $1.4 \mathrm{~nm}$ (AA, atomistic part of mixed system) for two component solvent-alanine systems. For the MARTINI potential the standard shift function was applied from $0.9 \mathrm{~nm}$ or $1.2 \mathrm{~nm}$ for cut-off values 1.2 and $1.4 \mathrm{~nm}$ respectively. For the hybrid two-component systems, the electrostatic potential was shifted between 0 and the cut-off distance, and a relative screening with $\varepsilon_{r}=15$ was applied in line with the standard screening used in MARTINI. The effect of changing $\varepsilon_{r}$ on the behavior of these systems was also evaluated.

In all simulations a leapfrog integrator with 2 fs time step was used with a neighbor list updated every 5 steps. Bond distances in atomistic molecules were constrained using the LINCS algorithm. ${ }^{33}$ Stochastic temperature coupling, ${ }^{34}$ with a time constant of $1.0 \mathrm{ps}$ and a reference temperature of $270 \mathrm{~K}$ (butane systems) or $320 \mathrm{~K}$ (systems containing water), was applied. A Berendsen barostat ${ }^{35}$ was used to equilibrate atomistic and hybrid two component systems at 1 bar before constant volume production runs. Most of the simulations were between 40-100 ns long. To equilibrate the two component systems consisting of butane and dialanine molecules, simulated annealing simulations (SA) were performed before the production runs. During the SA, the temperature was decreased linearly from 600 to $270 \mathrm{~K}$ over a period of $100 \mathrm{~ns}$.

\section{Coarse grained potentials}

The applicability of our hybrid model will depend on the compatibility between the CG and AA force fields. There is, however, no unique method to construct $\mathrm{CG}$ potentials from AA potentials. ${ }^{36}$ Even when the pair correlations are well described, other properties such as the pressure or solvation free energy will be off. In this work we tested four different methods to construct the CG potentials: the iterative
Boltzmann (IB) inversion method, ${ }^{37-39}$ the force matching (FM) method, ${ }^{40,41}$ a modification of the subtraction procedure (SB) originally described by Villa et al., ${ }^{42}$ and the MARTINI force field. ${ }^{32,43}$ In each case, the resolution of the coarse grained models is given by a 4 to 1 center of mass based mapping principle, in analogy to the standard mapping scheme used in the MARTINI model. A pressure correction is applied to the potentials to match the pressure of the reference atomistic system. Although the relation between pressure on the CG and AA level is non-trivial (for instance, one cannot simultaneously reproduce both pressure and compressibility ${ }^{44-46}$ ), we decide here on the pressure, in a similar way the adaptive resolution methods do, because we want to combine atomistic and CG models and they should have the same pressure. Details of the methods pertaining the current application are presented below.

Iterative Boltzmann inversion. In structure based coarse graining, a CG potential is determined in such a way that predefined target functions, which structurally characterize the system, are reproduced in the CG simulation. In the iterative Boltzmann inversion method ${ }^{37}$ radial distribution functions, $g_{\text {ref }}(r)$, are the target functions. The $g_{\text {ref }}(r)$ can be obtained either from experiment or from atomistic simulations. Through the simple Boltzmann inversion

$$
V_{\mathrm{PMF}}(r)=-k_{\mathrm{B}} T \ln g_{\text {ref }}(r),
$$

where $k_{\mathrm{B}}$ denotes the Boltzmann constant and $T$ the temperature, the potential of mean force $V_{\mathrm{PMF}}$ between pairs of CG particles can be obtained. Unfortunately, this potential of mean force cannot be directly used in a CG model, because part of it represents multibody contributions from all the particles in the system. Therefore, an iterative procedure should be used:

$$
V_{i+1}^{\mathrm{CG}}(r)=V_{i}^{\mathrm{CG}}+k_{\mathrm{B}} T \ln \left[\frac{g_{i}(r)}{g_{\mathrm{ref}}(r)}\right] .
$$

The procedure is initiated with the $V_{\mathrm{PMF}}$ potential from the simple Boltzmann inversion. The subscript ${ }_{i}$ denotes the iteration number. According to the Henderson ${ }^{47}$ theorem the IB method should give a unique two body CG interaction for the given $g_{\text {ref }}(r)$. However, in practice the convergence is difficult to achieve and varies for different parts of the potential. ${ }^{39,48}$ Especially attractive parts require an extensive number of iterations whereas repulsive parts converge relatively fast. Nevertheless, $g_{\text {ref }}(r)$ is reproduced with a good accuracy already after a small number of iterations. To enhance the convergence during the iteration procedure the CG potential can be modified to reproduce the target pressure. For example, a correction to the potential scaling linearly with the pressure difference can be applied ${ }^{49,50}$

$$
\Delta V(r)=A\left(1-\frac{r}{R_{c}}\right)
$$

where $R_{c}$ is a user defined cut off for the pair interaction potential and $A$ can be defined as

$$
A=-|(\Delta P)| k_{\mathrm{B}} T f
$$




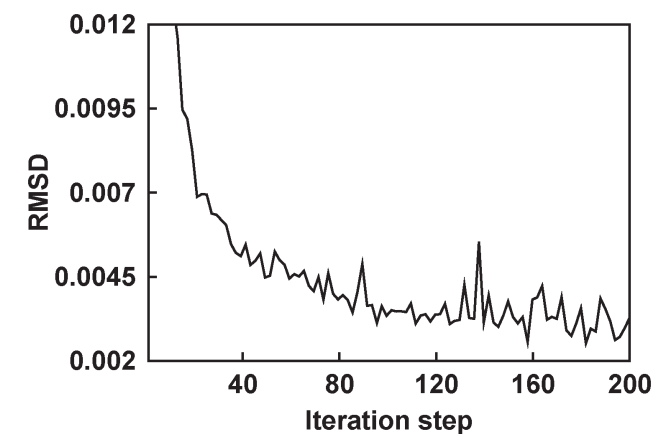

Fig. 2 Convergence of IB method for a system of liquid butane. The root mean square deviation (RMSD) between the calculated radial distribution function (RDF), $g(r)$, and the target RDF, $g_{\text {ref }}(r)$, is plotted as function of the number of iterations.

with $\Delta P$ being the pressure mismatch and $f$ a scaling factor.

To calculate the IB potentials in the current application, the VOTCA $^{50}$ package was used. From a 100 ns reference simulation of pure butane, the reference molecular radial distribution functions were generated. A 200 ps CG simulation was performed at each iteration. The pressure correction was applied at every cycle and the convergence was measured as the root mean square deviation (RMSD) $\sqrt{\frac{\sum\left(g(r)-g_{\text {ref }}(r)\right)^{2}}{n}}$ (where the summation runs over all distance bins $n$ ) between the reference and calculated radial distribution function. The convergence of the RMSD is shown in Fig. 2. After 175 iterations the obtained potential was converged and used in the subsequent calculations.

Force matching. Force matching ${ }^{40,41}$ is another technique to construct $\mathrm{CG}$ potentials. It also uses information from reference atomistic simulations to construct the effective CG interaction. Force matching does not rely on pair correlation functions, i.e. pair potentials of mean force, but matches forces on the $\mathrm{CG}$ interaction sites as close as possible with the forces at the AA level. Thus it aims at reproducing the multibody potential of mean force with a set of CG interaction functions, in the present case with effective pair interaction functions. ${ }^{41}$ In order to evaluate FM CG potentials, first reference forces on CG beads are calculated as a sum of atomistic forces $\mathbf{f}_{\gamma}$

$$
\mathbf{F}_{i}^{\text {ref }}=\sum_{\gamma} \mathbf{f}_{\gamma}, \forall \gamma \in N_{i}
$$

Note, eqn (6) is only correct for center of mass mapping as used in our hybrid method. Next a model is constructed in which the CG forcefield depends linearly on $\mathbf{M}$ parameters $g_{1}, \ldots, g_{M}$, here these are coefficients of cubic splines used to tabulate the $\mathrm{CG}$ forces. Subsequently a fitting procedure is performed, which in essence involves a solution of the following set of $N \times L$ equations:

$$
\mathbf{F}_{i l}^{c g}\left(g_{1}, \ldots, g_{m}\right)=\mathbf{F}_{i l}^{\mathrm{ref}}, i=1, \ldots, N, l=1, \ldots, L
$$

Here $N$ is the number of CG beads and $L$ the number of frames from the AA trajectory used for coarse graining. $L$ should be large enough to make the set of equations overdetermined. The calculation is usually repeated for a number of smaller parts of the trajectory and the final result is constructed as an average over the set of solutions, making the procedure less time consuming. Potentials are obtained by integrating tabulated force functions.

FM CG potentials were constructed using the VOTCA ${ }^{50}$ package. From a $100 \mathrm{~ns}$ reference simulation of pure butane (the same as used for the IB method) the reference forces were generated. For each force matching calculation 35 frames from the reference simulation were used. The final tabulated force function was calculated as an average of all functions fitted to the fragments of the atomistic trajectory. As the last step a pressure correction of the form presented in eqn (4) was iteratively applied, to tune the pressure to the reference atomistic value. Strictly speaking, this type of pressure correction is not formally correct in the case of force matching. Pressure correction for force matching does exist, ${ }^{45,51}$ it is however not yet implemented in the VOTCA package.

The subtraction method. To overcome sampling problems in dilute solutions, where $g(r)$ for solute-solute pairs converges very slowly, a new method was introduced recently by Villa and coworkers. ${ }^{42,52}$ In this approach, which we will call here the subtraction (SB) method, the PMF between two solute molecules $V_{\mathrm{PMF}}^{\mathrm{AA}}$ in a solvent box is calculated. Then, to remove the solvent contributions from the PMF the calculation is rerun without direct solute-solute interactions to obtain a correcting potential $V_{\mathrm{PMF} \text {,excl }}$. This $V_{\mathrm{PMF} \text {,excl }}$ is subtracted from the $V_{\mathrm{PMF}}^{\mathrm{AA}}$ and an effective two body solute-solute $\mathrm{CG}$ interaction is obtained:

$$
V_{\mathrm{SB}}^{\mathrm{CG}}=V_{\mathrm{PMF}}^{\mathrm{AA}}-V_{\mathrm{PMF}, \text { excl }}
$$

In the original SB method by Villa et al. this is done by computing $V_{\mathrm{PMF}, \mathrm{excl}}^{\mathrm{CG}}$ on the CG level. This means a PMF between two $\mathrm{CG}$ solute molecules is computed where the direct solute-solute interaction is turned off, while the interactions between the solute particles and the surrounding solvent and the solvent-solvent interactions are CG interactions that had previously been determined, for example by iterative Boltzmann inversion. Note that this subtraction method is not limited to IB. It just means one assumes a set of CG interactions in the environment of the solutes and subtracts the PMF between two noninteracting solutes in this environment from the atomistic target PMF. The resulting $V_{\mathrm{SB}}^{\mathrm{CG}}$ is an effective solute-solute pair interaction which if added to the set of $\mathrm{CG}$ environmental interactions (solvent-solvent and solute-solvent) reproduces the target solute-solute PMF (at infinite dilution). In the present case the subtraction method is modified to target at a CG-CG interaction that is particularly suited in the present hybrid approach. Therefore one subtracts the PMF between two noninteracting solute molecules in atomistic environment from the atomistic target PMF.

$$
V_{\mathrm{SB}}^{\mathrm{CG}}=V_{\mathrm{PMF}}^{\mathrm{AA}}-V_{\mathrm{PMF}, \text { excl }}^{\mathrm{AA}}
$$

Since this means one subtracts the atomistic multibody effects of the environment from the target PMF, this modification of the procedure permits to obtain an effective two body $V_{\mathrm{SB}}^{\mathrm{CG}} \mathrm{CG}$ interaction in an otherwise atomistic medium. 
To construct the $V_{\mathrm{PMF} \text {,excl }}^{\mathrm{AA}}$ component of the SB potential, a constraint method was applied:

$$
V_{\mathrm{PMF}}(r)=-\int_{r}^{r_{c}}\left[\left\langle f_{c}\right\rangle_{s}+\frac{2 k_{\mathrm{B}} T}{s}\right] \mathrm{d} s,
$$

where $\left\langle f_{c}\right\rangle$ is an average force exerted on two particles separated by a fixed distance $s$ and $r_{c}$ is the interaction cut-off. The second term in the integral removes entropic contributions, that are different for consecutive distances because of larger volumes sampled at larger separations. The LINCS method ${ }^{33}$ was used to fix the distances between the two evaluated molecules. The separation for constrained molecules was varied with $0.02 \mathrm{~nm}$ increment, from $0.22 \mathrm{~nm}$ up to $r_{c}$. The $V_{\mathrm{PMF}}^{\mathrm{AA}}$ component was obtained by simply inverting the RDF of the fully atomistic system according to eqn (2). The resulting $V_{\mathrm{SB}}^{\mathrm{CG}}$ potential was additionally pressure corrected to the reference pressure of 1 bar, using the iterative pressure correction procedure (eqn (4)).

The MARTINI force field. Unlike the methods introduced above the MARTINI force field does not focus on reproducing structural details at a particular state point for a specific system, rather the aim is the thermodynamic transferability between different solute environments. This is achieved by extensive calibration of the building blocks of this $\mathrm{CG}$ force field against thermodynamic data, in particular water oil partitioning coefficients, similar to the methodology used to parametrize the $53 \mathrm{a} 5 / 6$ set of the GROMOS force field. ${ }^{31}$ The distinct feature of the model is its simplicity, expressed through the use of standard interaction potentials and few parameters. Only four main bead types are defined: polar, non-polar, apolar and charged. Polar sites represent neutral groups of atoms that would easily dissolve in water, apolar sites represent hydrophobic moieties and non-polar groups represent mixed blocks, which are partly polar and partly apolar (e.g. propanol). Charged sites are reserved for ionized species. The Lennard-Jones potential is used to model nonbonded interactions

$$
V_{\mathrm{LJ}}(r)=4 \varepsilon_{i j}\left[\left(\frac{\sigma_{i j}}{r}\right)^{12}-\left(\frac{\sigma_{i j}}{r}\right)^{6}\right],
$$

where $i, j$ denote interaction sites, $\sigma_{i j}$ is the bead size and the strength of interaction is defined by $\varepsilon_{i j}$. In addition to the LJ interaction, charged groups interact via the standard electrostatic Coulombic potential. Both interactions are cutoff at a distance $1.2 \mathrm{~nm}$. To reduce the cutoff noise the $\mathrm{LJ}$ is shifted to zero between $0.9 \mathrm{~nm}$ and $1.2 \mathrm{~nm}$ in such a way that both potential and force vanish at the cutoff distance. ${ }^{53}$ The Coulombic interaction is also shifted, with the shift affecting the potential from $0.0 \mathrm{~nm}$ to $1.2 \mathrm{~nm}$. Shifting of the electrostatic potential mimics the effect of a distance dependent screening. Uniform dielectric screening $\varepsilon=15$ is additionally introduced, to compensate the lack of explicit screening in polar solvents. Bonded interactions are described with standard harmonic functionals. The original MARTINI forcefield parametrization was aimed for lipid and surfactant systems. ${ }^{43}$ The subsequent papers introduced parameters for peptides and proteins ${ }^{54}$ and carbohydrates. ${ }^{55}$
For the current aim of using the MARTINI model in hybrid simulations, the MARTINI potential for the liquid butane system was modified slightly to better represent the molecular $g_{\text {ref }}(r)$ from the reference atomistic simulation. Particularly, the bead size was increased by $0.25 \AA$ to match the position of the first peak in the $g_{\text {ref }}(r)$. Subsequently the value of $\varepsilon$ was increased by $5 \%$ to match the atomistic pressure. The modified potential will be named MARTINI* in the rest of the paper and the standard interaction, used in the peptide systems, will be referred to without the "*" sign.

\section{Analysis details}

Kirkwood-Buff integrals. To analyze the behavior of the systems in the hybrid representation we analyzed the preferential solvation parameters $\delta_{\mathrm{CG}}=G_{\mathrm{CG}, \mathrm{CG}}-G_{\mathrm{CG}, \mathrm{AA}}$ and $\delta_{\mathrm{AA}}=G_{\mathrm{AA}, \mathrm{AA}}-G_{\mathrm{CG}, \mathrm{AA}}$. These parameters are calculated using Kirkwood-Buff integrals: ${ }^{56}$

$$
G_{i, j}=4 \pi \int_{0}^{R_{\mathrm{cut}}}\left[g_{i, j}(r)-1\right] r^{2} \mathrm{~d} r
$$

where $g_{i, j}(r)$ is the radial distribution function between the centers of mass of molecule $i$ and $j$ and $R_{\text {cut }}$ is the distance beyond which the integrand vanishes. The calculated quantities are used to describe the affinities between molecules modeled at different resolutions and to predict (micro)phase separation.

Association constants. In order to assess strength of interactions between atomistic molecules in the hybrid model we calculate the association constant $K_{a}$, which are directly linked to the above Kirkwood-Buff integrals. $K_{a}$ is given as

$$
K_{a}=4 \pi \int_{0}^{R_{\text {cut }}} \exp \left(\frac{-V_{\mathrm{PMF}}}{k_{\mathrm{B}} T}\right) r^{2} \mathrm{~d} r
$$

with $V_{\mathrm{PMF}}$ denoting the potential of mean force between the two molecules and $R_{\text {cut }}$ being a specified cut-off distance.

Solvation free energy. Comparison of thermodynamic properties of the constructed CG potentials was assessed via the free energy of solvation $\Delta G$, which we calculated using the thermodynamic integration ${ }^{57}$ procedure. For each calculation 21 simulations of $1 \mathrm{~ns}$ length at equally spaced $\lambda$ values between 0 and 1 were performed. The last 800 ps were used for analysis. A soft core method was used to remove singularities during the calculations. Integration over the $\lambda$ variable was performed using a trapezoidal rule.

\section{Results and discussion}

\section{A Comparing CG potentials for butane}

To test the hybrid model we chose a simple system of liquid butane. The butane molecule, which is modeled as a chain of four united carbon atoms by the GROMOS forcefield, is represented by a single bead on the coarse grained level. In Fig. 3A CG potentials for the butane-butane interaction are shown. The CG potentials were obtained either with iterative Boltzmann inversion, force matching, or subtraction method, or with the MARTINI* model. Substantial differences between the potentials are apparent. Notably, IB and FM potentials are shallow and have significant interaction energy 

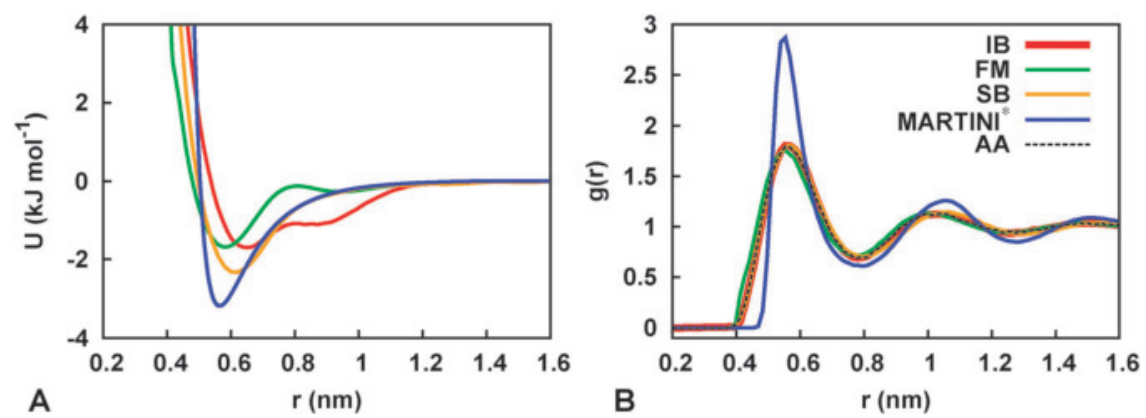

Fig. 3 Coarse-grained butane-butane potentials. In panel A, CG potentials constructed with the four methods are shown, namely iterative Boltzmann (IB, red), force matching (FM, green), subtraction method (SB, orange), and MARTINI* model (blue). Panel B displays the molecular radial distribution functions for liquid butane, simulated with each of the four CG potentials. A reference radial distribution function from a fully atomistic simulation (black dashed line) is also presented.

contributions from the second neighbors as evidenced by an additional repulsive region (FM) or long range attraction (IB). Furthermore both IB and FM potentials have a softer repulsive part at short distances compared to SB and particularly to the MARTINI* LJ potential. Both the MARTINI* and SB potentials are of comparable shape, especially considering the attractive region beyond $0.7 \mathrm{~nm}$. MARTINI* butanes are however more repulsive at short distances and have a slightly deeper minimum. In spite of these discrepancies, IB, FM and SB potentials, when employed in a coarse grained simulation of pure butane, generate a very similar $g(r)$ as shown in Fig. 3B. Note that this result is not trivial as the different methods to construct the $\mathrm{CG}$ potentials, while relying on the same atomistic reference sampling, have different targets. Only IB was optimized to reproduce the atomistic RDF. FM is optimized to reproduce a multibody PMF, that means it targets at the pair RDF, but at the same time also at higher order correlation functions and depending on the "basis set" of CG interaction functions (in this case pair potentials) has to make compromises as far as the pair RDF is concerned. The SB methods targets indeed at the pair RDF (in the form of the pair PMF) but since we do not iterate and do not subtract $V_{\mathrm{PMF}, \mathrm{IBcl}}^{\mathrm{IB}}$ but $V_{\mathrm{PMF} \text {,excl }}^{\mathrm{AA}}$ we do not by construction match the RDF. With the MARTINI* potential the phase in $g(r)$ is preserved but the amplitude is too high. This means that molecules are more ordered than in the reference atomistic liquid butane system, a consequence of using of a 12-6 Lennard-Jones interaction functional which results in a very steep repulsion at short distances.

Although structurally the IB, FM and SB potentials generate similar equilibrium ensembles, this does not imply that the thermodynamics is described equally well. To quantify this, we calculated the free energy of solvation $\Delta G$ (transfer of one molecule from vacuum into its solution) for each of the four models. In Table 1 the results are shown together with the average potential energies and results for the reference atomistic system. Regardless of the distinct $g(r)$ for MARTINI* with respect to the other potentials, $\Delta G$ for the MARTINI*, IB and SB potentials is very similar and close to the reference atomistic $\Delta G_{\text {ref. }}$ The $\Delta G$ obtained with the FM potential is substantially lower. As we will see later, this is a problem in hybrid simulations as it will result in preferential solvation effects.
Table 1 Free energy of solvation, $\Delta G$, of a CG butane molecule dissolved into its own bulk. Results obtained with each of the four CG potentials are shown, together with the free energy for an AA system. Association constants $K_{a}$ of two atomistic butane molecules dissolved in CG butane are also shown

\begin{tabular}{lll}
\hline Representation & $\Delta G ;(\mathrm{kJ} / \mathrm{mol})$ & $K_{a}$ \\
\hline AA, Gromos 53a5 & $-13.6 \pm 0.7$ & 17.1 \\
CG, IB & $-11.3 \pm 0.7$ & 18.6 \\
CG, FM & $-7.1 \pm 0.5$ & 18.3 \\
CG, MARTINI* & $-11.6 \pm 1.6$ & 16.6 \\
CG, SB & $-10.3 \pm 1.0$ & 17.3 \\
\hline
\end{tabular}

\section{B PMFs for AA butane in CG solvent}

In this section we examine the properties of hybrid systems simulated with the different CG potentials. To this aim we calculated potentials of mean force (PMFs) between two atomistic butane molecules solvated in coarse grained butane. The constraint method, described in section IIIC, was used to compute the PMFs. The setup for each experiment was identical, except for the interactions between $\mathrm{CG}-\mathrm{CG}$ and CG-AA pairs of molecules which were represented with either one of the four CG potentials (IB, FM, SB, and MARTINI*). Fig. 4A shows the resulting PMFs including a reference PMF calculated in a fully atomistic butane system. Neither of the CG approaches yields a hybrid model where the two atomistic molecules show the same pair interaction as if they were in a fully atomistic environment. Overall, the MARTINI* and SB PMFs resemble the reference PMF the most, although the attractive and repulsive regimes are more apparent in both cases. This effect is much more pronounced in case of the IB PMF. Atomistic molecules in the IB solvent are strongly attracted to each other at short distances but effectively repel themselves when outside the first solvation shell. The FM potential exhibits an enhanced attraction over the entire range of the calculated interaction. We can quantify the total interaction strength by means of the association constant $K_{a}$. Here we integrate the PMF up to a cutoff distance $R_{\text {cut }}$ of $1.6 \mathrm{~nm}$ to obtain $K_{a}$, using eqn (13). Results are summarized in Table 1. The association constants are similar for all four CG potentials. However, the calculated $K_{a}$ depends on the integration interval, and differences are larger when a smaller $R_{\text {cut }}$ is chosen (Fig. 4B), showing largest deviations for the IB and FM potentials. Since the target of the hybrid approach is 

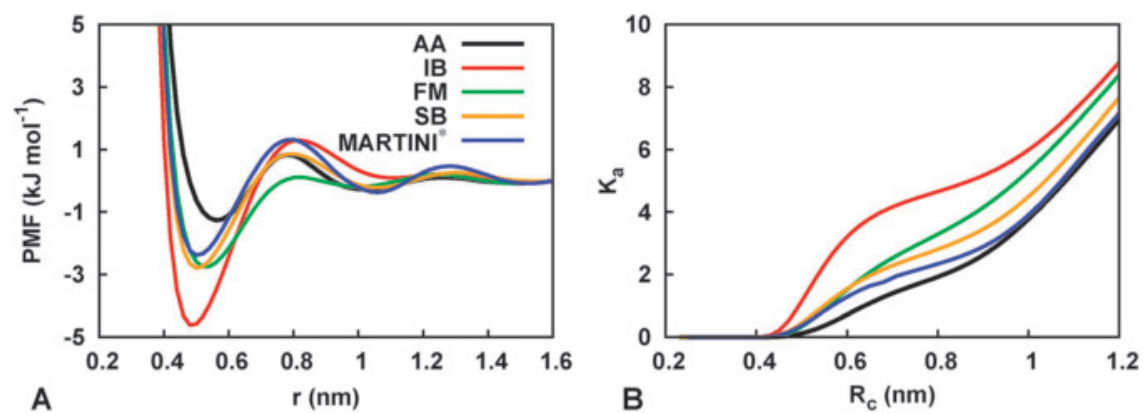

Fig. 4 Performance of different CG potentials in the hybrid model. Panel A: Potential of mean force between two AA butane molecules dissolved in CG butane. Panel B: Association constant as a function of integration cut-off for the two AA butane molecules. Results obtained for CG potentials constructed with the four methods are shown, namely iterative Boltzmann (IB, red), force matching (FM, green), subtraction method (SB, orange), and MARTINI* model (blue). Results for a reference atomistic systems are shown with a thick black line.

that the interaction strength between two atomistic molecules in the $\mathrm{CG}$ environment should reproduce the one in a fully atomistic system, it appears from these results that the SB and MARTINI* potentials are the most promising candidates for use in such a hybrid model. This assumption is further tested below.

\section{Preferential solvation in mixed AA/CG butane}

In order to confirm our observations from the potential of mean force calculations, we simulated liquid butane systems in which half of the butane molecules are represented as coarse grained particles and half of them atomistically. Equilibrium ensembles were generated with Langevin dynamics as described in the Methods section. As before, we performed calculations for all four CG potentials. To assess the mixing of the $\mathrm{AA}$ and $\mathrm{CG}$ butane molecules we computed the radial distribution functions $g_{i, j}(r)$ between AA-AA, AA-CG, and $\mathrm{CG}-\mathrm{CG}(i, j)$ butane pairs. The results are shown in Fig. 5A-D. In the ideal scenario molecules do not know the resolution of their neighbors and therefore do not have any preference of one representation above the other. Since we use the same CG potential for $\mathrm{CG}-\mathrm{CG}$ and $\mathrm{AA}-\mathrm{CG}$ pairs we would expect identical $g_{i, j}(r)$. That is not the case for IB and FM systems, where we see different preferences in the first and second shell or clustering of atomistic molecules, respectively. In the IB system atomistic molecules, which strongly attract each other on a short distance, favor neighbors of the same type. Interaction on the longer distance, however, is more favorable with the unlike type, since the CG potential is deeper in that position ( $c f$. Fig. 3A). This causes formation of transient structures, e.g. atomistic butane wires or small clusters. The clustering is even more pronounced in the FM system due to lower solvation free energy of AA butane in CG butane than in itself (Table 1).

The clustering effect can be quantified with preferential solvation parameters $\delta_{i}$ that we calculate by integrating $g_{i, j}(r)$ using eqn (12). In Table 2 the preferential solvation results are summarized. Indeed we can see an increased preferential solvation for alike molecules in the FM system. In the IB system this is not so clear, since Kirkwood-Buff integrals are calculated over three solvation shells and the differences are averaged out. Therefore we additionally computed preferential solvation parameters $\delta_{\mathrm{CG}}^{R \frac{1}{2}}$ using a cut-off $R_{\frac{1}{2}}=0.8 \mathrm{~nm}$.
Large positive numbers for the first shell (and large negative for the rest) support the distance dependent solvation preferences discussed above. In the MARTINI and SB systems the $\delta_{\mathrm{AA}}$ and $\delta_{\mathrm{CG}}$ values are much closer to zero, implying that there is no significant preferential solvation. This is also clear from the overlapping RDFs for $\mathrm{CG}-\mathrm{CG}$ and $\mathrm{CG}-\mathrm{AA}$ pairs (Fig. 5C and D).

In panel $\mathrm{E}$ of Fig. 5 the $g_{\mathrm{AA}, \mathrm{AA}}(r)$ from IB, FM, SB and MARTINI* hybrid systems are compared to $g_{\text {ref }}(r)$ obtained from a fully atomistic simulation. The RMSD of all four $g_{\mathrm{AA}, \mathrm{AA}}(r)$ to $g_{\text {ref }}(r)$ is shown in Table 2. A larger deviation is apparent for the IB potential, whereas the three other models show a similar agreement with the reference $g(r)$. Overall, the performance of the SB potential is considered best, since all $g_{i, j}(r)$ are very much alike (Fig. 5D). Additionally all of them resemble $g_{\text {ref }}(r)$. This would make the SB potential the optimal choice for the hybrid model. Nevertheless, in the MARTINI* hybrid system, where $g_{\mathrm{CG}, \mathrm{CG}}(r)$ and $g_{\mathrm{AA}, \mathrm{AA}}(r)$ are different, we do not observe preferential solvation and the atomistic part of the system reproduces the reference molecular radial distribution function $g_{\text {ref }}(r)$. That suggests that the thermodynamic accuracy of the CG potential, more than the structural one, is essential for the proper mixing of resolutions.

Although the structural and thermodynamic analysis presented above indicates that the hybrid model performs well, provided that a suitable CG potential is used, our analysis so far has mainly concerned the behavior at the CG level. Whether the atomistic degrees of freedom are also unperturbed is investigated next. In Fig. 5F, radial distribution functions for carbon atom pairs are presented, for the SB, MARTINI* hybrid systems as well as the purely atomistic reference system. We limited our analysis to the SB and MARTINI* potentials, since the performance of these potentials in the hybrid model is best. The discussion, however, is general and concerns all four cases. Despite the similar $g_{\mathrm{AA}, \mathrm{AA}}(r)$ and $g_{\text {ref }}(r)$ at the CG level ( $c f$. Fig. 5E), there is a noticeable overstructuring of the carbon pair correlation functions. This effect we attribute to the partly missing interactions of the carbon atoms with their coarse-grained neighbors, which could lead to a change in the alignment of the butane chains in atomistic surrounding compared to the CG (spherical particle) surrounding. As can be seen in Fig. 5F the effect is rather small; importantly the global structure is not affected ( $c f$. Fig. 5C and D). 

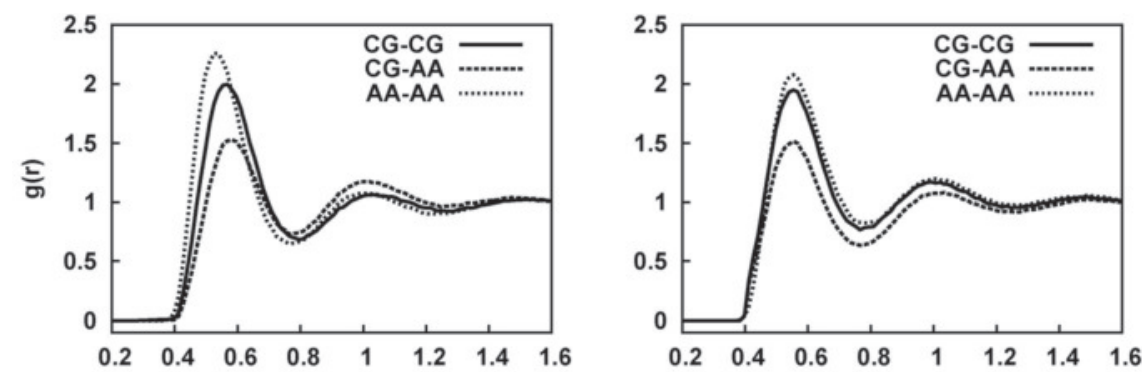

A:IB

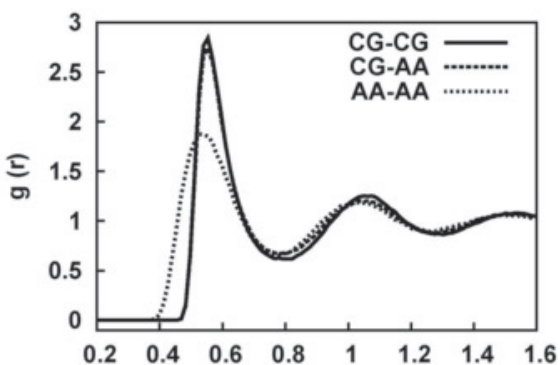

B:FM

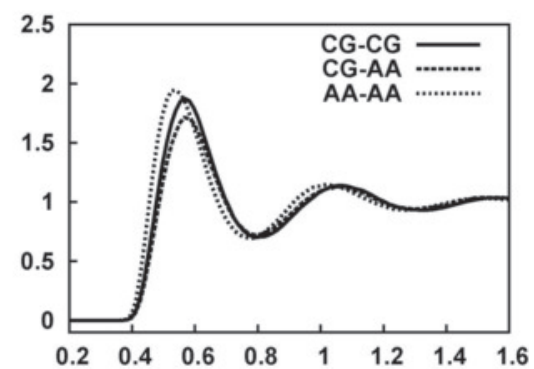

C:MARTINI*

D:SB
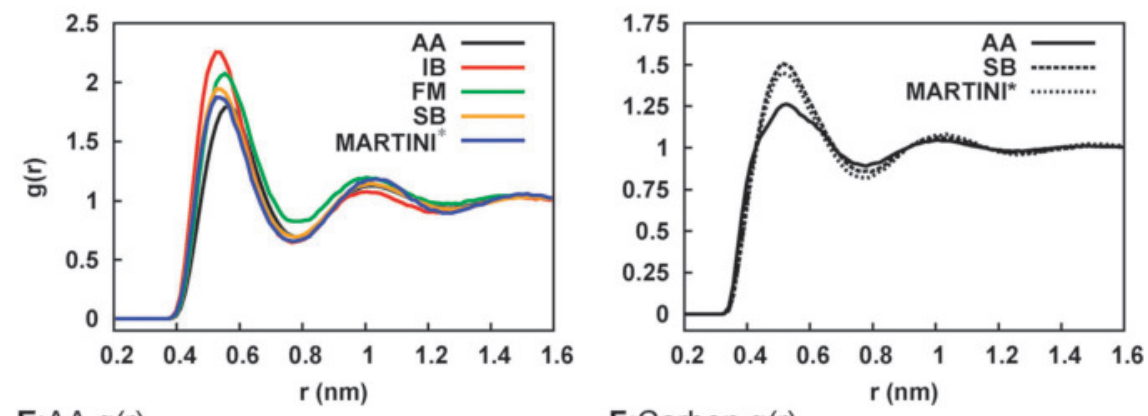

F:Carbon $\mathrm{g}(\mathrm{r})$

Fig. 5 Even mixture of AA and CG molecules. A,B,C,D panels: molecular radial distribution functions $g_{\mathrm{AA}, \mathrm{CG}}(r)$ (long dashed line), $g_{\mathrm{AA}, \mathrm{AA}}(r)$ (dotted line) and $g_{\mathrm{CG}, \mathrm{CG}}(r)$ (full line) for IB, FM, MARTINI* and SB potentials, respectively. In panel E atomistic molecular radial distribution functions $g_{\mathrm{AA}, \mathrm{AA}}(r)$ are shown. Black line represents $g_{\text {ref }}(r)$, red line: CG component is described with iterative Boltzmann inversion, green line: force matching, orange line: subtraction method, blue line: MARTINI* model. In panel F carbon radial distribution functions are presented for atomistic part of SB hybrid model (long dashed line), MARTINI* (dotted line) and reference atomistic simulation (full line).

Table 2 Preferential solvation parameters $\delta_{i}$ obtained from equal mixtures of AA and CG butane molecules. The CG part of the systems is represented with one of the four CG potentials. Results obtained with two cut-off distances, $R_{c}$ and $\frac{1}{2} R_{c}$, are presented. The last column shows the RMSD of the molecular $g(r)$ from the hybrid system to the molecular $g_{\text {ref }}(r)$ of a reference atomistic system

\begin{tabular}{lrrrrl}
\hline CG potential & \multicolumn{1}{c}{$\delta_{\mathrm{AA}}^{R_{c}}$} & \multicolumn{1}{c}{$\delta_{\mathrm{CG}}^{R_{c}}$} & $\delta_{\mathrm{AA}}^{\frac{1}{2} R_{c}}$ & $\delta_{\mathrm{CG}}^{\frac{1}{2} R_{c}}$ & RMSD \\
\hline IB & -118 & -111 & 220 & 202 & 0.182 \\
FM & 902 & 684 & 348 & 274 & 0.109 \\
SB & 61 & 8 & 49 & 69 & 0.101 \\
MARTINI* & -17 & -32 & -32 & -8 & 0.099 \\
\hline
\end{tabular}

\section{Association of AA dialanine in CG solvents}

In addition to the tests performed on pure liquid butane here we validate the mixed model on two component systems composed of dialanine dissolved either in liquid butane or in water. These uncommon systems were chosen here first because of higher complexity than the pure butane case: each time there are two CG bead types and two distinct $\mathrm{CG}$ interaction potentials. Moreover, Coulombic interactions are introduced between atomistic molecules. Second we would like to know, if the MARTINI forcefield, without any modifications, could be used as a default CG representation in hybrid simulations. Standard MARTINI potentials are used to represent effective interactions between butanebutane, butane-alanine and alanine-water pairs of molecules. The dialanine molecule is described by two beads on the CG level and therefore two virtual sites are constructed, each representing the center of mass of the single alanine aminoacid. The butane molecule, as previously, is represented with one CG bead. Four atomistic water molecules are also represented by a single $\mathrm{CG}$ bead. All interactions between dialanine molecules are computed atomistically. For the electrostatic interactions, it is not clear beforehand whether or not a screening of the charges should be applied. In fully atomistic 

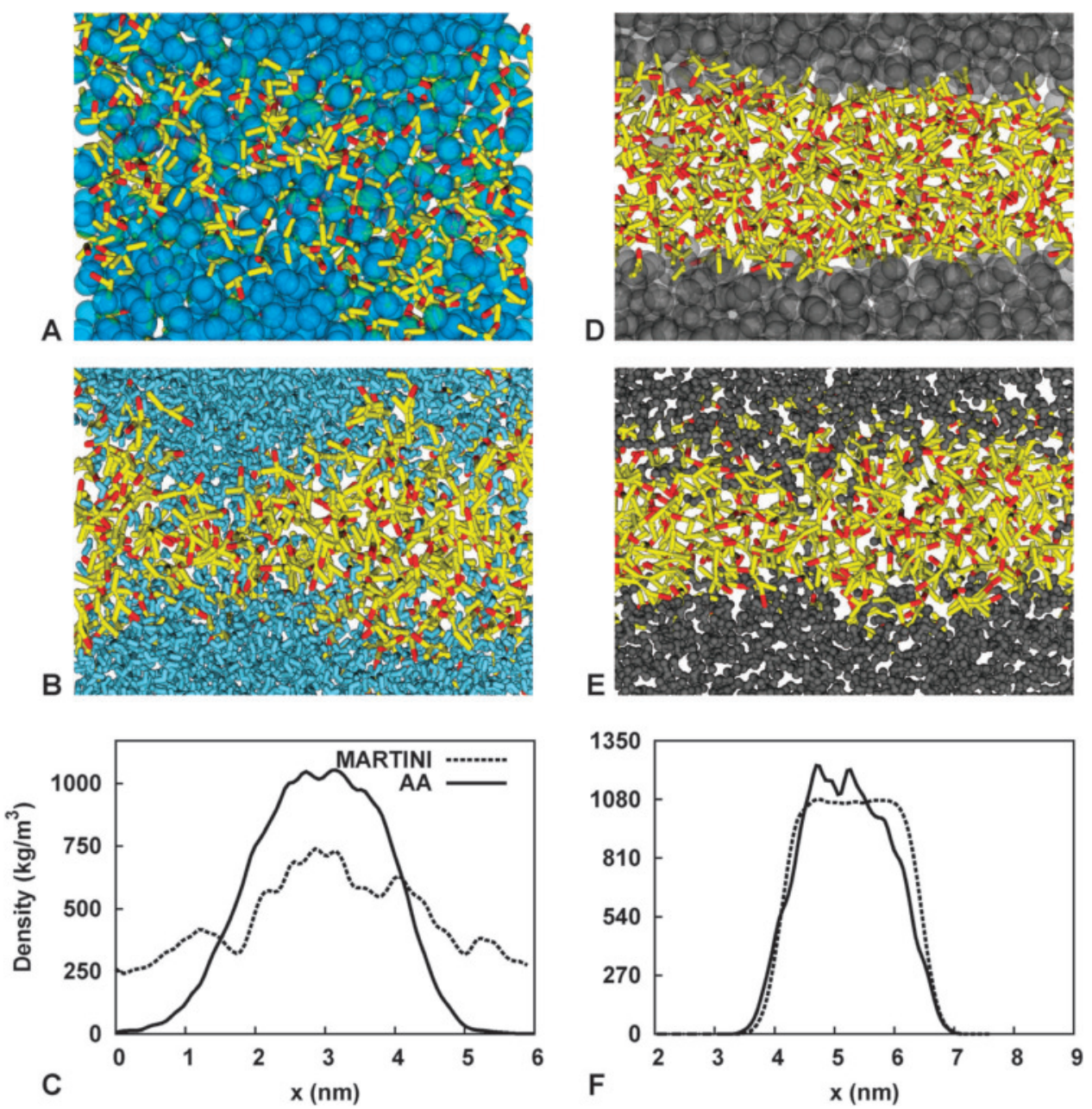

Fig. 6 Mixing of atomistic dialanine peptides in coarse-grained solvents. The solvent is either water (A,B,C) or butane (D,E,F). Snapshots from an atomistic simulation (A,D) are compared to MARTINI hybrid simulations (B,E). Butane is depicted as grey spheres, water as blue spheres, and the peptides with yellow carbon and red oxygen atoms. Panels $\mathrm{C}$ and $\mathrm{F}$ show the density profiles of the dialanine peptides for atomistic (full line) and hybrid (long dashed line) systems.

systems, such a screening is not necessary as the solvent performs the screening explicitly. In the MARTINI model, however, most solvents (including water) are modeled as a simple LJ fluid lacking electrostatic interactions. Coulombic interactions are therefore scaled with a uniform dielectric constant $\varepsilon=15$ to account for the absence of explicit screening. Here we tested both approaches, i.e. either screening the atomistic peptide-peptide interactions with $\varepsilon_{r}=15$, or no $\left(\varepsilon_{r}=1\right)$ or limited $\left(\varepsilon_{r}=2\right)$ screening. For reference, fully atomistic systems were also simulated. See section IIIB for details.

With butane as solvent, clear phase separation is observed between butane and dialanine, as illustrated in Fig. 6D and E. The density of the peptides across the box is shown in Fig. 6F. The width of the layer and the density of dialanine molecules inside the layer are similar between the hybrid and fully atomistic systems. Also the sharpness of the interface is comparable in both cases. The results shown in Fig. 6D-F pertain the situation in which the electrostatic interactions were screened with $\varepsilon_{r}=15$; similar results were obtained in the absence of screening. Replacing the apolar butane solvent by a polar solvent like water, we also observe phase separation (Fig. 6A-C) in both atomistic and hybrid models. However, in this case phase separation is only observed with unscreened electrostatics and mixing occurs for $\varepsilon_{r}=15$ (not shown). Even with unscreened electrostatic interactions, the phase separation in the hybrid model is not as pronounced as in the fully atomistic system (Fig. 6C). We also calculated the PMF for two atomistic dialanine peptides in $\mathrm{CG}$ solvent. Results are shown in Fig. 7A for butane as a solvent, and Fig. 7B for water. In a qualitative sense, the hybrid model reproduces the effect of the solvent on the peptide-peptide interaction: an apolar medium like butane strongly promotes dimerization, whereas in the aqueous environment the interaction strength is at the level of $k T$. However, it turns out that the alanine-alanine interactions are too weak in the hybrid system when using the standard screening $\varepsilon_{r}=15$ of the MARTINI model. This is apparent from the comparison of the hybrid PMF to that of the fully atomistic reference system. In the case of butane, this screening is way too strong. Better performance is obtained lowering the dielectric constant to a value between 1 and 2 (Fig. 7A). In case of water, lowering of 

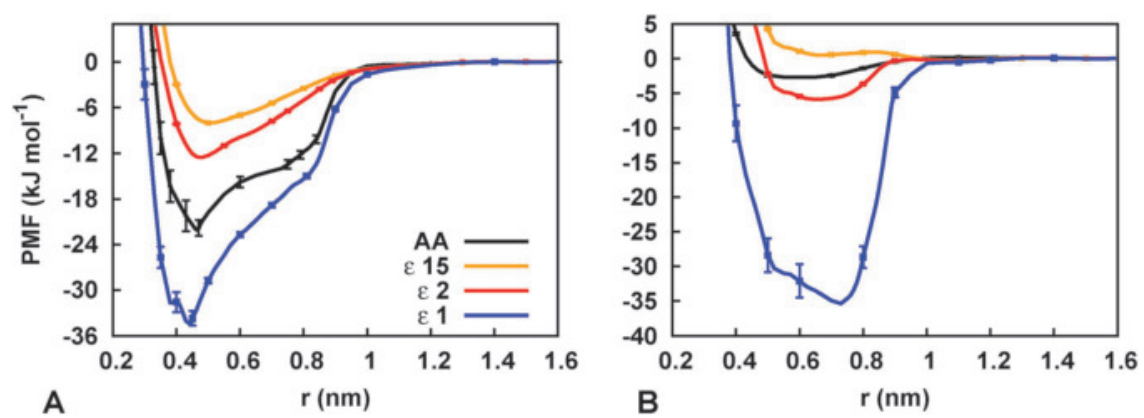

Fig. 7 Potential of mean force between two atomistic dialanines in CG solvent using MARTINI. Results for butane are shown in panel A, for water in panel B. The effect of different screening constants $\varepsilon_{r}=1,2$, and 15, is included. The reference atomistic system is shown with a solid line.

the dielectric constant also improves the results with the best agreement to the reference potential at a value in the range between 4 to 6 .

\section{Summary and conclusions}

In this work we designed and tested a straightforward hybrid model to bridge atomistic and coarse grained representations of an arbitrary system. We focused our efforts on the coarse grained description, assuming it to be a crucial element for the hybrid model to function. Three procedures to construct CG potentials were tested: iterative Boltzmann (IB) inversion, force matching (FM), and a subtraction (SB) method. In addition, the pre-parameterized MARTINI force field was used. We simulated two systems to explore the performance of the hybrid model: pure liquid butane molecules and dialanine peptides dissolved either in an apolar solvent, butane, or a polar solvent, water.

Using the first system, i.e. pure butane, we showed that only with MARTINI and SB CG potentials both structural and thermodynamic properties of the hybrid system remain close to those of a fully atomistic reference system. For these potentials, no preferential solvation effects were observed implying that the molecules do not know their resolution, mixing equally well with both $\mathrm{AA}$ and $\mathrm{CG}$ neighbors. The potentials obtained with IB or FM are less effective, causing distinct preferential solvation effects. We do not claim that better potentials could not be obtained also with IB and FM approaches (for example by applying additional thermodynamic constraints during the parameterization), however, two ingredients of the CG potential appear crucial: (i) reproducing the solvation free energy of the atomistic model, and (ii) a correct balance between short range and long range forces. In case of the FM potential, the solvation free energy is too low, and in case of the IB potential an additional repulsive regime is present. Both lead to non-ideal mixing of the AA and CG molecules. An accurate representation of the structural pair correlations is not essential, as can be concluded from the good performance of the MARTINI potential. Small deviations of the intra-bead atomistic degrees of freedom are observed irrespective of the CG potential used. It seems unavoidable in this kind of hybrid simulations that the internal atomistic degrees of freedom are somewhat perturbed, especially for systems with a low density of AA particles. Butane may be a worst-case scenario, however, due to its small size. Overall, the best results are obtained with the SB method that was recently developed by Villa et al. ${ }^{42,52}$ The SB CG potentials show an accurate energy of solvation, correct representation of the pair correlation functions, and well-balanced overall shape of the potential, which results in the lack of preferential solvation effects in the hybrid simulations. Whether this is generally the case might depend on the complexity of the multibody interactions present in the system. An indication that butane is possibly less complicated than other systems is that IB, FM, and SB yield very similar pair RDFs. This would be very different, e.g., for water due to important 3-body interactions. Further tests on parameterizing the optimal CG potentials for our hybrid approach are clearly required. Extended discussion about potentials similar to the $\mathrm{SB}$ potentials can be additionally found in the recent work by Brini et al. ${ }^{58}$

Advantage of the use of the MARTINI force field is that MARTINI potentials are readily available for a large range of biomolecular systems. ${ }^{43,54,55} \mathrm{We}$ used the second system, i.e. dialanine peptides mixed with either butane or water, to test how well the hybrid MARTINI model performs with more complicated cases involving electrostatics. From calculations of the PMF between two atomistic dialanine peptides in CG solvent, we conclude that the effect of the solvent is captured in a qualitative sense; the peptides are attractive in the apolar solvent and are rather neutral in polar solvent. Better quantitative agreement with the full atomistic system is obtained when the dielectric constant is chosen as a free parameter. For the apolar butane solvent, best matching is obtained with $\varepsilon_{r}$ between 1 and 2, for water at a value around 5. However, in the case of the $1: 1$ mixture of dialanine and water, a screening of 1 is required to reproduce the phase separation seen in the fully atomistic system. More testing is required to see whether these values are generically valid, and how the effective screening depends on the density of CG versus AA sites. Preliminary results from our group indicate that atomistic water (in the form of bundled $\mathrm{SPC}^{59}$ ) and $\mathrm{CG}$ MARTINI water mix homogeneously when using a dielectric constant of 2 . Nevertheless, even with the standard $\varepsilon_{r}=15$ value of the MARTINI force field, we showed here that a system in which a large amount of dialanine peptides is mixed with butane phase separates with the layer thickness and density of molecules the same in atomistic and hybrid representations. In an aqueous environment, the use of the recently parameterized polarizable MARTINI water model ${ }^{60}$ might improve the 
performance of the hybrid model as it is capable of explicit screening.

Overall we conclude that the proposed hybrid scheme appears to work, at least for the simple systems considered so far. We are currently exploring its range of applications, considering for instance the binding of an enzyme to a protein with only the binding pocket and ligand in full atomic detail, or the association of proteins in which only the surface of the protein is modeled in full detail.

\section{Acknowledgements}

A.R. acknowledges HPC-Europe2 program for sponsoring a visit to the Polymer Theory Group, MPI-Mainz, VOTCA developers Christoph Junghans and Victor Rühle for their kind help, Victor Rühle for careful reading of the manuscript, and Alex de Vries for useful discussions. Funding through the TOP-program of the Netherlands Organisation for Scientific Research (NWO) is also acknowledged.

\section{References}

1 W. F. van Gunsteren, D. Bakowies, R. Baron, I. Chandrasekhar, M. Christen, X. Daura, P. Gee, D. P. Geerke, A. Glaettli, P. H. Hünenberger, M. A. Kastenholz, C. Oostenbrink, M. Schenk, D. Trzesniak, N. F. A. van der Vegt and H. B. Yu, Biomolecular modeling: Goals, problems, perspectives, Angew. Chem., Int. Ed., 2006, 45, 4064

2 M. Karplus and J. A. McCammon, Molecular dynamics simulations of biomolecules, Nat. Struct. Biol., 2002, 9, 646.

3 S. J. Marrink, A. H. de Vries and D. P. Tieleman, Lipids on the move: Simulations of membrane pores, domains, stalks and curves, Biochim. Biophys. Acta, Biomembr., 2009, 1788, 149.

4 Coarse-Graining of Condensed Phase and Biomolecular Systems, ed. G. A. Voth, Taylor and Francis, Inc., 2008.

5 G. S. Ayton, W. G. Noid and G. A. Voth, Multiscale modeling of biomolecular systems: in serial and in parallel, Curr. Opin. Struct. Biol., 2007, 17, 192.

6 C. Peter and K. Kremer, Multiscale simulation of soft matter systems - from the atomistic to the coarse-grained level and back, Soft Matter, 2009, 5, 4357.

7 T. Murtola, A. Bunker, I. Vattulainen, M. Deserno and M. Karttunen, Multiscale modeling of emergent materials: biological and soft matter, Phys. Chem. Chem. Phys., 2009, 11, 1869.

8 C. Clementi, Coarse-grained models of protein folding: toy models or predictive tools?, Curr. Opin. Struct. Biol., 2008, 18, 10.

9 W. Tschöp, K. Kremer, O. Hahn, J. Batoulis and T. Bürger, Simulation of polymer melts. II. From coarse-grained models back to atomistic description, Acta Polym., 1998, 49, 75.

10 G. Milano and F. Müller-Plathe, Mapping atomistic simulations to mesoscopic models: A systematic coarse-graining procedure for vinyl polymer chains, J. Phys. Chem. B, 2005, 109, 18609.

11 B. Hess, S. Leon, N. F. A. van der Vegt and K. Kremer, Long time atomistic trajectories from coarse grained simulations: Bisphenol-a polycarbonate, Soft Matter, 2006, 2, 409.

12 V. A. Harmandaris, N. P. Adhikari, N. F. A. van der Vegt and K. Kremer, Hierarchical modeling of polystyrene: From atomistic to coarse-grained simulations, Macromolecules, 2006, 39, 6708

13 A. Y. Shih, P. L. Freddolino, S. G. Sligar and K. Schulten, Disassembly of nanodiscs with cholate, Nano Lett., 2007, 7, 1692.

14 A. P. Heath, L. E. Kavraki and C. Clementi, From coarse-grain to all-atom: Toward multiscale analysis of protein landscapes, Proteins: Struct., Funct., Bioinf., 2007, 68, 646.

15 T. Carpenter, P. J. Bond, S. Khalid and M. S. P. Sansom, Self-assembly of a simple membrane protein: Coarse-grained molecular dynamics simulations of the influenza M2 channel, Biophys. J., 2008, 95, 3790 .
16 A. Villa, C. Peter and N. F. A. van der Vegt, Self-assembling dipeptides: conformational sampling in solvent-free coarse-grained simulation, Phys. Chem. Chem. Phys., 2009, 11, 2077.

17 A. J. Rzepiela, L. V. Schäfer, N. Goga, H. J. Risselada, A. H. de Vries and S. J. Marrink, Reconstruction of atomistic details from coarse-grained structures, J. Comp. Chem., 2010, 31, 1333.

18 B. Ensing, S. O. Nielsen, P. B. Moore, M. L. Klein and M. Parrinello, Energy conservation in adaptive hybrid atomistic/ coarse-grain molecular dynamics, J. Chem. Theory Comput., 2007, 3, 1100 .

19 M. Praprotnik, L. Delle Site and K. Kremer, Adaptive resolution molecular-dynamics simulation: Changing the degrees of freedom on the fly, J. Chem. Phys., 2005, 123, 224106.

20 A. Heyden and D. G. Truhlar, Conservativealgorithm for an adaptive change of resolution in mixed atomistic/coarse-grained multiscale simulations, J. Chem. Theory Comput., 2008, 4, 217.

21 M. Christen and W. F. van Gunsteren, Multigraining: An algorithm for simultaneous fine-grained and coarse-grained simulation of molecular systems, J. Chem. Phys., 2006, 124, 154106.

22 P. Liu and G. A. Voth, Smart resolution replica exchange: An efficient algorithm for exploring complex energy landscapes, J. Chem. Phys., 2007, 126, 045106.

23 E. Lyman, F. M. Ytreberg and D. M. Zuckerman, Resolution exchange simulation, Phys. Rev. Lett., 2006, 96, 28105.

24 A. Warshel and M. Levitt, Theoretical studies of enzymic reactions: dielectric, electrostatic and steric stabilization of the carbonium ion in the reaction of lysozyme, J. Mol. Biol., 1976, 103, 227.

25 J. Michel, M. Orsi and J. W. Essex, Prediction of partition coefficients by multiscale hybrid atomic-level/coarse-grain simulations, J. Phys. Chem. B, 2008, 112, 657.

26 Q. Shi, S. Izvekov and G. A. Voth, Mixed atomistic and coarse-grained molecular dynamics: Simulation of a membranebound ion channel, J. Phys. Chem. B, 2006, 110, 15045.

27 M. Neri, C. Anselmi, M. Cascella, A. Maritan and P. Carloni, Coarse-grained model of proteins incorporating atomistic detail of the active site, Phys. Rev. Lett., 2005, 95, 218102.

28 S. Nielsen, R. Bulo, P. Moore and B. Ensing, Recent progress in adaptive multiscale molecular dynamics simulations of soft matter, Phys. Chem. Chem. Phys., 2010, 12, 12401.

29 M. Praprotnik, K. Kremer and L. Delle Site, Adaptive molecular resolution via a continuous change of the phase space dimensionality, Phys. Rev. E, 2007, 75.

30 B. Hess, C. Kutzner, D. van der Spoel and E. Lindahl, GROMACS 4: Algorithms for highly efficient, load-balanced, and scalable molecular simulation, J. Chem. Theory Comput., 2008, 4, 435

31 C. Oostenbrink, A. Villa, A. E. Mark and W. F. van Gunsteren, A biomolecular force field based on the free enthalpy of hydration and solvation: the GROMOS force-field parameter sets 53A5 and 53A6, J. Comput. Chem., 2004, 25, 1656.

32 S. J. Marrink, H. J. Risselada, S. Yefimov, D. P. Tieleman and A. H. de Vries, The MARTINI force field: Coarse grained model for biomolecular simulations, J. Phys. Chem. B, 2007, 111, 7812 .

33 B. Hess, H. Bekker, H. J. C. Berendsen and J. G. M. Fraaije, Lincs: A linear constraint solver for molecular simulations, J. Comput. Chem., 1997, 18, 1463.

34 W. F. van Gunsteren and H. J. C. Berendsen, A leap-frog algorithm for stochastic dynamics, Mol. Simul., 1988, 1, 173.

35 H. J. C. Berendsen, J. P. M. Postma, W. F. van Gunsteren, A. D. Nola and J. R. Haak, Molecular dynamics with coupling to an external bath, J. Chem. Phys., 1984, 81, 3684.

36 A. A. Louis, Beware of density dependent pair potentials, J. Phys.: Condens. Matter, 2002, 14, 9187.

37 A. K. Soper, Empirical potential monte carlo simulation of fluid structure, Chem. Phys., 1996, 202, 295.

38 A. P. Lyubartsev and A. Laaksonen, Calculation of effective interaction potentials from radial distribution functions: A reverse monte carlo approach, Phys. Rev. E: Stat. Phys., Plasmas, Fluids, Relat. Interdiscip. Top., 1995, 52, 3730.

39 D. Reith, M. Pütz and F. Müller-Plathe, Deriving effective mesoscale potentials from atomistic simulations, J. Comput. Chem., 2003, 24, 1624 
40 S. Izvekov, M. Parrinello, C. J. Burnham and G. A. Voth, Effective force fields for condensed phase systems from ab initio molecular dynamics simulation: a new method for force-matching, J. Chem. Phys., 2004, 120, 10896.

41 W. G. Noid, J. W. Chu, G. S. Ayton, V. Krishna, S. Izvekov, G. A. Voth, A. Das and H. C. Andersen, The multiscale coarse-graining method. I. A rigorous bridge between atomistic and coarse-grained models, J. Chem. Phys., 2008, 128, 244114.

42 A. Villa, N. F. A. van der Vegt and C. Peter, Self-assembling dipeptides: including solvent degrees of freedom in a coarsegrained model, Phys. Chem. Chem. Phys., 2009, 11, 2068.

43 S. J. Marrink, A. H. de Vries and A. E. Mark, Coarse grained model for semiquantitative lipid simulations, J. Phys. Chem. B, 2004, 108, 750 .

44 H. Wang, C. Junghans and K. Kremer, Comparative atomistic and coarse-grained study of water: What do we lose by coarsegraining?, Eur. Phys. J. E, 2009, 28, 221.

45 S. Izvekov, P. W. Chung and B. M. Rice, The multiscale coarse-graining method: Assessing its accuracy and introducing density dependent coarse-grain potentials, J. Chem. Phys., 2010, 133, 064109.

46 M. E. Johnson, T. Head-Gordon and A. A. Louis, Representability problems for coarse-grained water potentials, J. Chem. Phys., 2007, 126, 144509.

47 R. L. Henderson, A uniqueness theorem for fluid pair correlation functions, Phys. Lett. A, 1974, 49, 197.

48 S. Jain, S. Garde and S. K. Kumar, Do inverse monte carlo algorithms yield thermodynamically consistent interaction potentials?, Ind. Eng. Chem. Res., 2006, 45, 5614.

49 F. Müller-Plathe, Coarse-graining in polymer simulation: from the atomistic to the mesoscopic scale and back, Chem. Phys. Chem, 2002, 3, 754.

50 V. Rühle, C. Junghans, A. Lukyanov, K. Kremer and D. Andrienko, Versatile object-oriented toolkit for coarse-graining applications, J. Chem. Theory Comput., 2009, 5, 3211.
51 A. Das and H. C. Andersen, The multiscale coarse-graining method. V. Isothermal-isobaric ensemble, J. Chem. Phys., 2010, 132, 164106.

52 A. Villa, C. Peter and N. F. A. van der Vegt, Transferability of nonbonded interaction potentials for coarse-grained simulations: Benzene in water, J. Chem. Theory Comput., 2010, 6, 2434 .

53 D. van der Spoel, E. Lindahl, B. Hess, A. R. van Buuren, E. Apol, P. J. Meulenhoff, D. P. Tieleman, A. L. T. M. Sijbers, K. A. Feenstra, R. van Drunen and H. J. C. Berendsen, Gromacs User Manual version 4.0, 2005, www.gromacs.org.

54 L. Monticelli, S. K. Kandasamy, X. Periole, R. G. Larson, D. P. Tieleman and S. J. Marrink, The MARTINI coarse grained forcefield: Extension to proteins, J. Chem. Theory Comput., 2008, 4, 819 .

55 C. A. López, A. J. Rzepiela, A. H. de Vries, L. Dijkhuizen, P. H. Hünenberger and S. J. Marrink, The MARTINI coarse-grained force field: Extension to carbohydrates, J. Chem. Theory Comput., 2009, 5, 3195.

56 A. Ben-Naim, Molecular Theory of Solutions, Oxford University Press, USA, 2006.

57 W. F. van Gunsteren and H. J. C. Berendsen, Thermodynamic cycle integration by computer simulation as a tool for obtaining free energy differences in molecular chemistry, J. Comput.-Aided Mol. Des., 1987, 1, 171.

58 E. Brini, V. Marcon and N. F. A. van der Vegt, Conditional reversible work method for molecular coarse graining applications, Phys. Chem. Chem. Phys., 2011, DOI: 10.1039/ C0CP02888F.

59 M. Fuhrmans, B. P. Sanders, S. J. Marrink and A. H. de Vries, Effects of bundling on the properties of the SPC water model, Theor. Chem. Acc., 2010, 125, 335.

60 S. O. Yesylevskyy, L. V. Schäfer, D. Sengupta and S. J. Marrink, Polarizable water model for the coarse-grained MARTINI force field, PLoS Comput. Biol., 2010, 6, e1000810. 